\title{
UTILIZAÇÃO DO PROCESSO ANALÍTICO HIERÁRQUICO PARA AVALIAR OPERADORES LABORATORIAIS NA ANÁLISE DE FERRO*
}

\section{Resumo}

Rossana Bezerra de Azevedo Vasconcelos Seabra de Melo ${ }^{1}$ Carla Schwengber ten Caten ${ }^{2}$ Pablo Jordano Sacramento Mendes ${ }^{3}$ Daniela Sedraz Silva de Freitas ${ }^{4}$

O presente estudo apresenta um método de avaliação de desempenho de operadores na análise de ferro via úmida baseada na Norma ISO 2597-2, utilizando dados de carta de controle do processo como fonte de informação. A partir do Processo Analítico Hierárquico (AHP), definiram-se pesos que refletem o desempenho dos operadores quanto à produtividade, precisão e veracidade, indicadores estes que são combinados posteriormente num indicador geral de desempenho (IGD). Dados da carta de controle do Laboratório Químico do Terminal Marítimo de Ponta da Madeira (TMPM) foram utilizados para alimentar o IGD e gerar uma classificação dos operadores, possibilitando tomadas de decisão, tais como a dedicação exclusiva dos melhores operadores na análise de ferro e a realocação dos demais para outras atividades, e a identificação dos pontos fracos de cada operador com inferior desempenho, de forma a direcionar capacitações. Além destas melhorias diretas, verificou-se a possibilidade de utilizar 0 desempenho no IGD como critério para promoções dos operadores, servindo estas como incentivo para a equipe na busca contínua de melhoria em sua habilidade analítica, assim como o aumento da produtividade.

Palavras-chave: Análise de minério de ferro; Processo analítico hierárquico; Indicador de desempenho; Produtividade; Precisão e veracidade.

\section{THE USE OF ANALYTIC HIERARCHY PROCESS TO EVALUATE LABORATORY OPERATORS IN THE ANALYSIS OF IRON}

\section{Abstract}

This study presents a method for evaluating the performance of operators in the wet chemical analysis of iron based on ISO 2597-2, using process control chart data as a source of information. Using the Analytic Hierarchy Process (AHP) weights were defined in order to demonstrate the operators performance in terms of productivity, precision, and accuracy. These indicators are then combined into an overall performance indicator (IGD). Process control chart data obtained at the Chemical Laboratory of the Ponta da Madeira Port (TMPM) were used as input to the IGD. The objective was to obtain a ranking of operators, enabling decision-making on issues such as devoting the best operators to the iron analysis and relocation the remaining to other activities, and identification of operators' weaknesses in order to target training. In addition to this direct improvement, there is the possibility of using the IGD as a criterion for promoting operators aiming at boosting continuous improvement in their analytical skills, as well as increasing productivity.

Keywords: Wet analysis of iron; Analytic hierarchy process; Performance indicator; Productivity; Precision and accuracy.

Eng. Química, Eng. Química, Resp. Técnica por laboratório do Sistema Norte, Vale, São Luís, MA, Brasil.

2 Dra. Eng. de Materiais, Msc Eng. Produção, Vice Diretora da Escola de Engenharia, UFRGS, Porto Alegre, RS, Brasil.

3 Administrador, Gerente de Área de Desenvolvimento, Otimização de Processo e Laboratórios, Vale, Carajás, PA, Brasil.

4 Eng. Química, Resp. Técnica por laboratório do Sistema Norte, Vale, Carajás, PA, Brasil.

* Contribuição técnica ao 44 Seminário de Redução de Minério de Ferro e Matérias-primas, $15^{\circ}$ Simpósio Brasileiro de Minério de Ferro e 2o Simpósio Brasileiro de Aglomeração de Minério de Ferro, 15 a 18 de setembro de 2014, Belo Horizonte, MG, Brasil. 


\section{INTRODUÇÃO}

Em um ambiente competitivo, faz-se necessário criar ferramentas de avaliação do processo e das pessoas por ele responsáveis, de forma a medir seu desempenho e dar subsídio para implantação de melhorias contínuas, na busca pela excelência. A melhoria da qualidade, representada pela redução da variabilidade do processo, promove um aumento de produtividade, à medida que aumenta a probabilidade de geração de itens conformes. A melhoria da qualidade também leva a uma diminuição de custos, devido à redução do retrabalho, erros e atrasos, e da melhor utilização da tecnologia e matéria-prima. Conseqüentemente, a produtividade aumenta.

Mais especificamente no controle de qualidade de empresas mineradoras, faz-se necessário garantir a confiabilidade dos resultados analisados por não se tratar de um controle de qualidade de produto beneficiado, mas sim, de analisar com exatidão o teor de ferro e demais contaminantes do minério para dar subsídio a tomadas de ação estratégicas para empresa.

$\mathrm{Na}$ indústria de minério de ferro, este é analisado durante:

- Prospecção: onde amostras de pesquisa, comumente chamadas de amostras de geologia, são de áreas ainda não lavradas, com o objetivo de conhecer teor de $\mathrm{Fe}$, contaminantes e traços ali existentes de forma a dimensionar o valor da mina e consequente, valor das ações nas bolsas de valores, planejamento de lavras futuras, etc.

- Produção: oriundos da usina de beneficiamento podendo ser as de alimentação da usina também chamadas de Run of Mine (ROM) e os produtos propriamente ditos, após beneficiamento.

- Expedição: produto carregado nos trens para serem destinado ao porto para exportação.

- Embarque: material carregado em navios para exportação, sendo o teor de ferro analisado o principal parâmetro que define o faturamento de cada carga e consequentemente de toda empresa.

Notadamente, atenção especial deve ser dada a análise do teor de ferro em minério de ferro. A determinação de seu teor é baseada na Norma ISO 2597-2. Essa análise depende da percepção visual do operador na determinação do ponto final de cada uma das etapas de titulação, o que reforça a necessidade de avaliar pessoas quanto à veracidade e precisão'. Além destes dois parâmetros, sob o ponto de vista de gestão, é interessante avaliar os operadores quanto à produtividade.

Os processos de medição de laboratórios de minério são monitorados por meio da análise de materiais de referencia (MR) juntamente com cada batelada de análises de amostras de minério. $O$ valor analisado deste MR é inserido em carta de controle cujos limites de controle são estabelecidos com base no desvio recomendado pela norma ou desvios do próprio processo de medição do laboratório em questão.

O presente estudo tem como objetivo utilizar informações do monitoramento do processo para medir o desempenho das pessoas na análise de ferro via úmida de um laboratório pertencente a uma empresa mineradora.

\footnotetext{
1 Veracidade: Grau de concordância entre a média de um número infinito de valores medidos repetidos e um valor de referência.

Precisão: Grau de concordância entre indicações ou valores medidos, obtidos por medições repetidas, no mesmo objeto ou em objetos similares, sob condições especificadas.
}

\footnotetext{
* Contribuição técnica ao 44ํㅗㄴ Seminário de Redução de Minério de Ferro e Matérias-primas, 15ำ Simpósio Brasileiro de Minério de Ferro e 2o Simpósio Brasileiro de Aglomeração de Minério de Ferro, 15 a 18 de setembro de 2014, Belo Horizonte, MG, Brasil.
} 
A análise minuciosa de dados de carta de controle, estratificada por pessoas, fornece informações valiosas para gestão do processo e gestão de pessoas, por possibilitar identificação de boas práticas, a identificação de oportunidade de melhoria, direcionamento a capacitações de forma equalizar método de análise, avaliar produtividade e, consequentemente, melhorar a qualidade do serviço analítico prestado.

O trabalho está estruturado em cinco seções, incluindo a presente introdução. A seção 2 apresenta o referencial teórico sobre a análise de ferro e conceitos sobre a forma de utilizar o método de AHP. A seção 3 apresenta o método utilizado e a seção 4, os resultados e discussões. Na seção 5 estão as conclusões do estudo, bem como a aplicação dos resultados em benefício do laboratório.

\subsection{Revisão da Literatura}

\subsubsection{Análise de ferro}

A análise do teor de Ferro por via úmida costuma ser feita com base na Norma 2597-2 - Minérios de ferro - Determinação do teor de ferro total Parte 2: Métodos de titulação após redução de cloreto de titânio (III).

Para a determinação deste teor, utiliza-se dicromato de potássio como titulante depois da redução do ferro (III) pelo cloreto de estanho (II) e cloreto de titânio (III). O excesso de agente redutor é então oxidado pelo dicromato de potássio diluído.

$\mathrm{Na}$ batelada de ensaios executados na rotina da empresa analisada no estudo de caso, três amostras de um mesmo padrão certificado são utilizadas para calcular o fator da Solução $\mathrm{K}_{2} \mathrm{Cr}_{2} \mathrm{O}_{7} 0,1 \mathrm{~N}$, através da fórmula:

$$
f=\frac{\% F e \times m}{V}
$$

Onde:

$f=$ Fator da solução de $\mathrm{K}_{2} \mathrm{Cr}_{2} \mathrm{O}_{7} 0,1 \mathrm{~N}$

$\% \mathrm{Fe}=$ Valor certificado do padrão de fatoração

$\mathrm{m}=$ massa da amostra

$\mathrm{V}=$ volume gasto na titulação

O acompanhamento do processo é feito analisando um MR, juntamente com cada batelada de análises executada. $O$ valor analisado deste padrão é inserido em carta de controle, cujos limites são estabelecidos com base no desvio recomendado pela norma. Calcula-se o \% Fe das demais amostras com a garantia que estão com Erro de Medida dentro da amplitude de aceite ou não, diante do resultado analisado do padrão de acompanhamento, utilizando a fórmula:

$$
\% F e=\frac{f \times V}{m}
$$

Caso o MR analisado tenha seu valor além dos limites de controle, toda a batelada é desconsiderada e repetida.

A aplicação deste método de análise via úmida é recomendado pela Norma na faixa de concentração de $30 \%$ a $72 \%$ de ferro em concentrados e aglomerados de minério de ferro. O item 8.2.1 desta Norma especifica ser 0,055\% o desvio padrão recomendado, dentro do mesmo laboratório, para análise de ferro utilizando decomposição ácida. 


\subsubsection{Processo analítico hierárquico (AHP)}

O AHP (Analytic Hierarchy Process ou Processo Analítico Hierárquico) é um método baseado em matemática e psicologia, desenvolvido na década de 1970 pelo Prof. Thomas Saaty.

Este método é utilizado para auxiliar na escolha ou tomada de decisões. Trata-se de um procedimento compreensivo e racional para estruturar um problema, para representar e quantificar seus elementos, para relacionar estes elementos com as metas globais e para avaliar soluções alternativas. É utilizado em todo o mundo, nas mais diferentes aplicações e áreas de atuação.

Em linhas gerais, a aplicação do método segue os seguintes passos operacionais:

- Decompor o problema de decisão em uma hierarquia de subproblemas mais facilmente compreendidos, sendo que cada um pode ser analisado independentemente.

- Os elementos da hierarquia podem relacionar-se com qualquer aspecto do problema de decisão - tangível ou intangível, podendo ser medidos com precisão ou estimados.

- Avaliar sistematicamente seus vários elementos, comparando-os um ao outro, em pares. As comparações podem usar dados concretos sobre os elementos, ou julgamentos sobre seu significado relativo ou a importância.

- Derivar as prioridades numéricas para cada uma das alternativas, com a avaliação crítica quanto à representatividade que estes números têm em relação às alternativas que irão guiar a tomada de decisão ou avaliação final.

\section{MATERIAIS E MÉTODOS}

O objetivo desta análise é ranquear os operadores de um laboratório da empresa Vale (Maranhão) para que se busque melhoria contínua na análise de ferro via úmida com produtividade satisfatória. A cada batelada de análise de ferro executada no laboratório, analisa-se uma amostra de MR de minério de ferro. $O$ dado desta análise é lançado em carta de controle que avalia se o resultado está dentro dos limites de controle. Caso não esteja, a batelada é descartada e as análises de todas as amostras são refeitas. $O$ operador que executou a análise registra seu nome e os dados da análise na carta de controle.

A partir destes dados históricos, foi possível avaliar: (i) a tendência ${ }^{2}$ de cada operador, calculada através da diferença entre a média dos resultados analisados por cada operador e o valor certificado do MR; (ii) a precisão de cada operador, analisando o desvio padrão dos resultados (embora não se tenha o mesmo número de medidas por operador, esta forma de medição é suficiente para gerar a informação necessária para compará-los); e (iii) a frequência das análises.

A produtividade de cada operador não foi efetivamente medida neste trabalho. Contabilizou-se o número de análises executadas por analista apenas como indicativo de qual está sendo a frequência de análise de ferro via, medida indireta de quanto cada um está sendo naturalmente selecionado a executar esta atividade em detrimento das demais, tendo mais oportunidade de desenvolver destreza em sua realização.

\footnotetext{
2 Tendência: Estimativa de um erro sistemático.

Erro sistemático: Componente do erro de medição que, em medições repetidas, permanece constante ou varia de maneira previsível.
}

\footnotetext{
* Contribuição técnica ao 44 Seminário de Redução de Minério de Ferro e Matérias-primas, 15ํ Simpósio Brasileiro de Minério de Ferro e 2ํ Simpósio Brasileiro de Aglomeração de Minério de Ferro, 15 a 18 de setembro de 2014, Belo Horizonte, ${ }_{1156}$ MG, Brasil.
} 
Para definição dos pesos que refletem o desempenho dos operadores quanto à produtividade, precisão e veracidade (que, combinados, irão gerar o IGD), utilizou-se o método AHP. A coleta de dados foi feita através de um questionário respondido por profissionais de diferentes laboratórios de minério de ferro.

Ao responder o questionário, cada colaborador opinou sobre o quanto um dos elementos é mais importante que os demais, aos pares. Para tanto, utilizou-se a escala de comparação proposta por Saaty, apresentada na Tabela 1.

Tabela 1 - Escala de comparação proposta por Saaty com adaptações

\begin{tabular}{|c|l|}
\hline Valor & Descriçăo \\
\hline 1 & Elemento i e elemento j tem igual importancia \\
\hline 3 & Elemento i é um pouco mais importante que o elemento $\mathrm{j}$ \\
5 & Elemento i é mais importante que o elemento $\mathrm{j}$ \\
7 & Elemento i é muito mais importante que o elemento j \\
9 & Elemento i é absolutamente mais importante que o elemento j \\
\hline $1 / 3$ & Elemento i é um pouco menos importante que o elemento $\mathrm{j}$ \\
$1 / 5$ & Elemento i é menos importante que o elemento j \\
$1 / 7$ & Elemento i é muito menos importante que o elemento $\mathrm{j}$ \\
$1 / 9$ & Elemento i é absolutamente menos importante que o elemento $\mathrm{j}$ \\
\hline
\end{tabular}

Respondidos os questionários, avaliou-se a consistência de cada entrevistado, Verificou-se, também, se havia opiniões que divergiam da maioria, de forma a obter consenso entre as opiniões. Tratados os dados espúrios, a média das opiniões foi utilizada como critério (peso) para cada um dos indicadores. Os três indicadores primários foram convertidos em uma pontuação de zero a cinco (Tabela 3), por já ser uma escala bem disseminada na empresa (Tabela 2).

Tabela 2 - Escala de níveis de desempenho: 0 a 5

\begin{tabular}{l|l}
\hline Nível 0 & Resultado abaixo do mínimo esperado \\
\hline Nível 1 ou Mínimo & Representa o resultado mínimo esperado \\
\hline Nível 2 ou Intermediário & Resultado entre o mínimo e o resultado esperado \\
\hline Nível 3 ou Meta & Representa o resultado esperado \\
\hline Nível 4 ou Superação & Resultado acima do nível esperado \\
\hline Nível 5 ou Excepcional & Representa excelência, resultados muito acima do esperado.
\end{tabular}

Tabela 3 - Conversão de indicadores primários em escala de níveis de desempenho 0 a 5

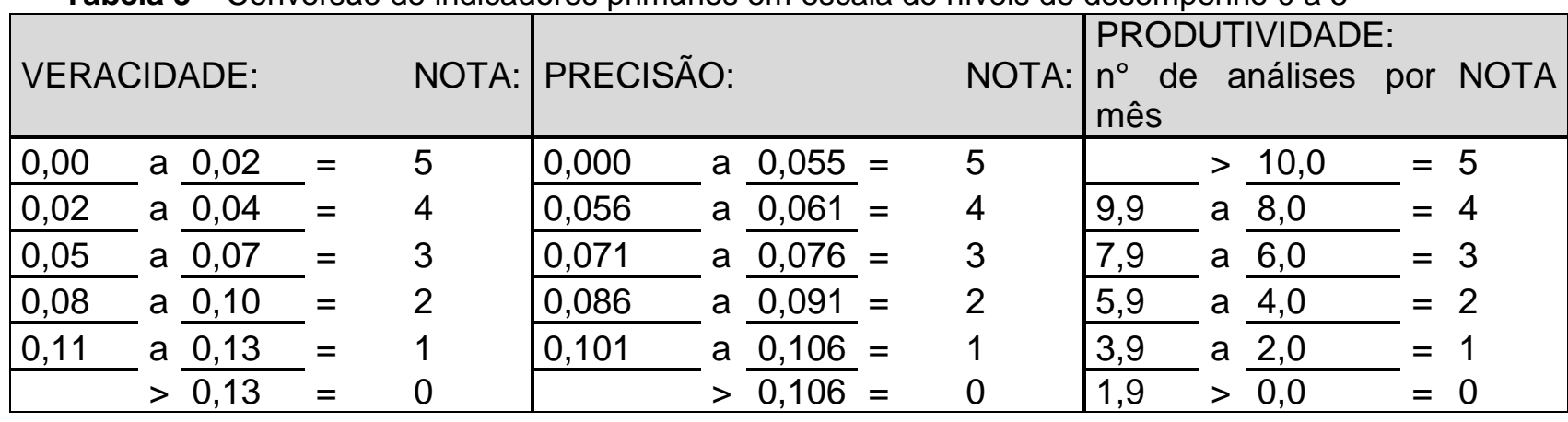

As faixas para cada um dos indicadores primários foram definidas de forma heurística a fim de obter os três indicadores primários em uma única base. Os pesos dos indicadores primários obtidos por meio do AHP são aplicados e gera-se o IDG, que possibilita avaliar individualmente o desempenho de cada operador e classificálos. 


\section{RESULTADOS E DISCUSSÃO}

Conforme apresentado na seção 3 , a definição dos pesos que refletem 0 desempenho dos operadores quanto à produtividade, precisão e veracidade, se deu por meio de avaliação das respostas de um questionário (Vide Anexo A). Oito profissionais de diferentes laboratórios de minério de ferro avaliaram os indicadores comparativamente. A partir da matriz de comparação das respostas do questionário, foram obtidos os pesos de importância dos indicadores (Tabela 4).

Tabela 4 - Matriz de comparações dos resultados e pesos de indicadores primários

\begin{tabular}{|l|c|c|c|c|}
\cline { 2 - 5 } \multicolumn{1}{c|}{} & PRECISÃO & VERACIDADE & PRODUTIVIDADE & PESOS \\
\hline PRECISÃO & 1 & $11 / 2$ & $51 / 2$ & 0,54 \\
\hline VERACIDADE & $2 / 3$ & 1 & $33 / 4$ & 0,36 \\
\hline PRODUTIVIDADE & $1 / 5$ & $1 / 4$ & 1 & 0,10 \\
\hline
\end{tabular}

O IGD utiliza os indicadores primários através da seguinte expressão: IGD = 0,54 X NÍVEL DE PRECISÂOO + 0,36 X NÍVEL DE EXATIDÃO + 0,1 X NÍVEL DE PRODUTIVIDADE Os 13 operadores do laboratório foram avaliados e ranqueados, conforme apresentado na Tabela 5.

Tabela 5 - Classificação de Operadores no IGD na Análise de Ferro Via Úmida

\begin{tabular}{|c|c|c|}
\hline Ranking & Pontuação & Operador \\
\hline $1^{\circ} \underline{0}$ & 3,80 & 2 \\
\hline $2^{\underline{0}}$ & 3,44 & 3 \\
\hline $3^{\circ}$ & 3,20 & 12 \\
\hline $4^{\circ}$ & 2,98 & 5 \\
\hline $5^{\circ}$ & 2,90 & 6 \\
\hline $6^{\circ}$ & 2,90 & 10 \\
\hline $7^{\circ}$ & 2,84 & 11 \\
\hline $8^{\circ}$ & 2,54 & 9 \\
\hline $9^{\circ}$ & 2,08 & 1 \\
\hline $1^{\circ}$ & 2,00 & 13 \\
\hline $11^{\circ}$ & 1,36 & 4 \\
\hline $12^{\circ}$ & 1,18 & 7 \\
\hline $13^{\circ}$ & 1,18 & 8 \\
\hline
\end{tabular}

De posse destes resultados, estabeleceu-se que os operadores com melhor desempenho ficassem dedicados à análise de ferro, enquanto que os de desempenho inferior ficassem dedicados às demais análises.

A partir desta definição, que ocorreu em 2012, observou-se melhoria de precisão nas análises da ordem de 8\% em relação a 2011.

Em termos práticos, esta melhoria impactou na minimização de incerteza no faturamento da empresa. 

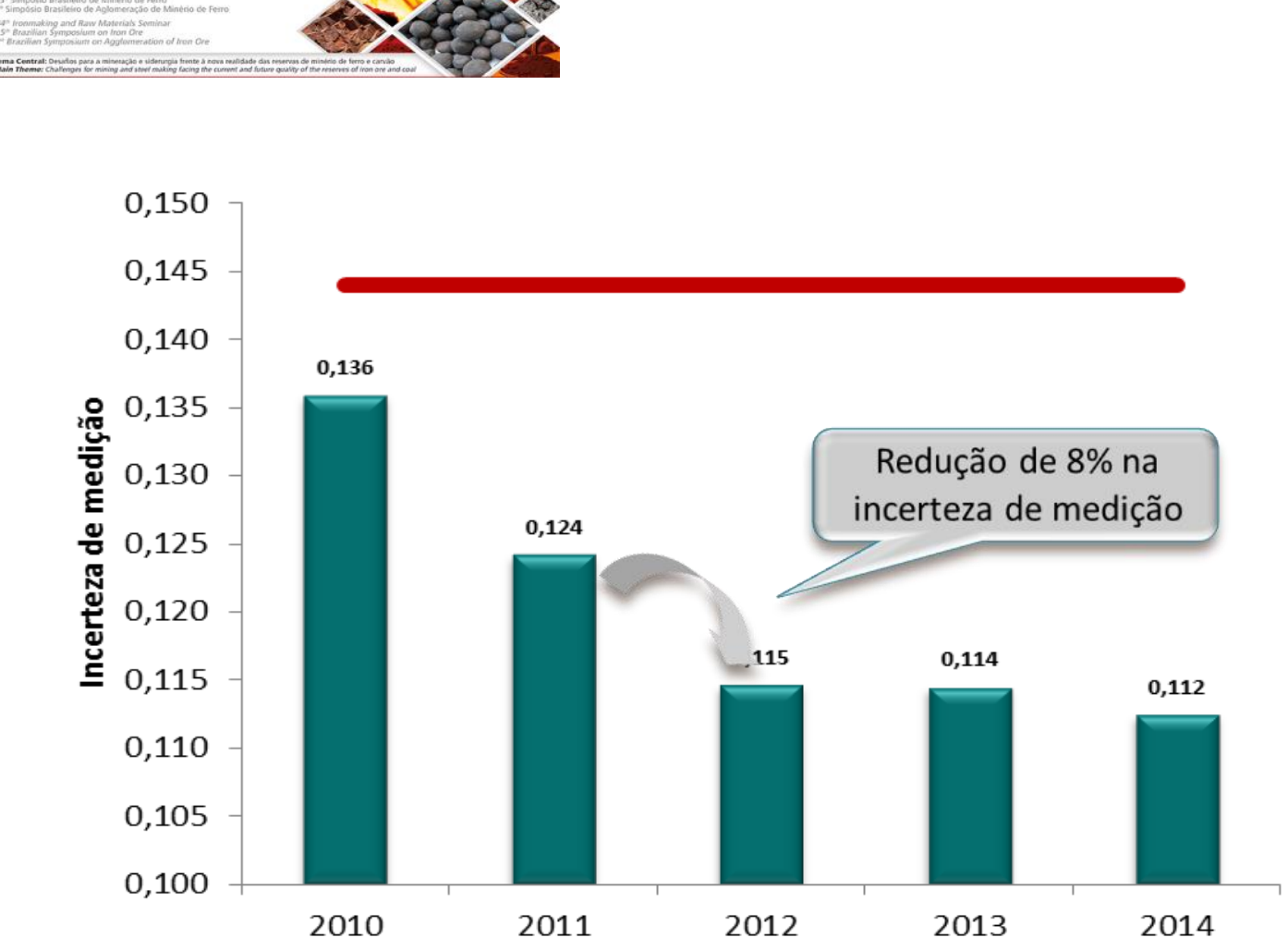

Incerteza para intervalo de confiança de 95\%

Limite aceitável para intervalo de confiança de 95\% (TC 103) = 0,144

Figura 1: Gráfico da evolução de desempenho em incerteza de medição na análise de ferro por via úmida.

\section{CONCLUSÕES}

Avaliando-se o resultado do indicador secundário IGD e dos indicadores primários que o compõe, foi possível identificar os pontos fortes e fracos de cada operador do laboratório de análise de ferro e tomar ações para melhoria do sistema de medição.

Criou-se uma escala de atividades por turno onde houve o direcionamento dos operadores com melhor desempenho no IGD na análise de ferro para executar preferencialmente esta análise. Os demais operadores foram direcionados para outras atividades.

Com a identificação dos pontos fracos de cada operador (desempenho inferior a três em um dos três indicadores primários) foi possível direcionar a capacitação e/ou orientação individualizada, baseada nas boas práticas observadas nos operadores de melhor desempenho.

Com a melhoria de precisão de análises, alcançada com as mudanças implantadas provenientes deste trabalho, houve minimização de incerteza no faturamento da empresa.

Um subproduto deste trabalho foi obter a opinião de diferentes profissionais em relação ao grau de importância que é dado aos indicadores de Precisão, Veracidade e Produtividade. A partir de relatos de alguns destes profissionais, conclui-se que esta informação foi necessária para se iniciar a avaliação do indicador, mas que estes pesos devem ser reavaliados de forma dinâmica, para refletirem a maturação da equipe ao longo do tempo.

* Contribuição técnica ao 44ํㅗㄴ Seminário de Redução de Minério de Ferro e Matérias-primas, 15ํ Simpósio Brasileiro de Minério de Ferro e 2o Simpósio Brasileiro de Aglomeração de Minério de Ferro, 15 a 18 de setembro de 2014, Belo Horizonte, MG, Brasil. 


\section{BIBLIOGRAFIA}

1 Costa HG. Auxílio Multicritério à Decisão: Método AHP. Latec/Universidade Federal Fluminense - Associação Brasileira de Engenharia de Produção (ABEPRO), Rio de Janeiro, 2006.

2 Falconi CV. TQC Controle da Qualidade Total (No Estilo Japonês), 1a.ed. Belo Horizonte: Fundação Cristiano Ottoni. 1992. 229p.

3 FALCONI CV. Gerenciamento Pelas Diretrizes (Hoshin Kanry). 2ª ed. Belo Horizonte: Fundação Cristiano Ottoni, 1996. 334p.

4 ISO 2597-2: Minério de ferro - Determinação do Teor de Ferro Total - Parte 2.

5 ISO 9001: Sistemas de gestão da qualidade - Requisitos.

6 ISO 17025: Requerimentos gerais para Laboratórios de Ensaio e Calibração.

7 Kardec A, Flores J, Seixas E. Gestão Estratégica e Indicadores de Desempenho. 1ª .ed. Rio de Janeiro: Qualitymark, 2002. 98p.

8 Montgomery D. Introdução ao controle estatístico da qualidade. 4ª ed. LTC- Livros Técnicos Científicos, 2004.

9 Ribeiro JLD, Ten Caten CS. Série Monográfica Qualidade: Controle Estatístico do Processo. Porto Alegre: FEENG/UFRGS, 2010.

10 Tadachi N., Flores MCX. Indicadores da Qualidade e do Desempenho. 1ª.ed. Rio de Janeiro: Qualitymark, 1997. 100p.

11 VIM - 2008 - Vocabulário Internacional de Metrológica.

12 Deming W. Edwards. Qualidade a revolução da administração. Rio de Janeiro: Marques Saraiva, 1990. 


\section{ANEXO - A \\ QUESTIONÁRIO DE AVALIAÇÃO DE CRITÉRIOS DE AVALIAÇÃO DE OPERADORE NA ANÁLISE DE FERRO VIA ÚMIDA}

ENTREVISTADO:

FORMAÇÃO:

FUNÇÃO:

DATA:

INSTRUÇÃO: Deve-se avaliar os três elementos a seguir, comparando-os um ao outro, em pares de acordo com a escala apresentada:

Elemento i X Elemento j (Antes de responder veja Escala de Respostas e Definições:

$$
\begin{aligned}
& \text { PRECISÃO X VERACIDADE }=>(） \\
& \text { PRECISÃO X PRODUTIVIDADE }=>(） \\
& \text { PRODUTIVIDADE X VERACIDADE }=>(）
\end{aligned}
$$

\begin{tabular}{|c|c|c|c|}
\hline Valor & Descrição & Valor & Descrição \\
\hline 1 & $\begin{array}{l}\text { Elemento i e elemento j têm igual } \\
\text { importância }\end{array}$ & & \\
\hline 3 & $\begin{array}{l}\text { Elemento i é um pouco mais importante } \\
\text { que o elemento j }\end{array}$ & $1 / 3$ & $\begin{array}{l}\text { Elemento jé um pouco mais } \\
\text { importante que o elemento i }\end{array}$ \\
\hline 5 & $\begin{array}{l}\text { Elemento i é mais importante que o } \\
\text { elemento j }\end{array}$ & $1 / 5$ & $\begin{array}{l}\text { Elemento j é mais importante que o } \\
\text { elemento i }\end{array}$ \\
\hline 7 & $\begin{array}{l}\text { Elemento i é muito mais importante que o } \\
\text { elemento j }\end{array}$ & $1 / 7$ & $\begin{array}{l}\text { Elemento j é muito mais importante } \\
\text { que o elemento } \mathrm{i}\end{array}$ \\
\hline 9 & $\begin{array}{l}\text { Elemento i é absolutamente mais } \\
\text { importante que o elemento j }\end{array}$ & $1 / 9$ & $\begin{array}{l}\text { Elemento j é absolutamente mais } \\
\text { importante que o elemento i }\end{array}$ \\
\hline
\end{tabular}

\section{ESCALA DE RESPOSTAS}

\section{DEFINIÇÕES}

\begin{tabular}{|l|l|}
\hline VERACIDADE: & $\begin{array}{l}\text { Ter veracidade é ter os resultados analisados centrados na média, mesmo } \\
\text { que com desvio alto entre as medidas. }\end{array}$ \\
\hline PRECISÃO: & $\begin{array}{l}\text { Ter precisão é ter o desvio pequeno, ter capacidade de reproduzir os } \\
\text { resultados. }\end{array}$ \\
\hline PRODUTIVIADE: & Quantas análises de ferro teve a iniciativa de fazer no ano \\
\hline
\end{tabular}

* Contribuição técnica ao 44 Seminário de Redução de Minério de Ferro e Matérias-primas, $15^{\circ}$ Simpósio Brasileiro de Minério de Ferro e 2o Simpósio Brasileiro de Aglomeração de Minério de Ferro, 15 a 18 de setembro de 2014, Belo Horizonte, MG, Brasil. 